\title{
Design of Artillery Operational Experiment Platform Based on the Information System Jianli Zhang ${ }^{1, a}$, Zhongwei Guo ${ }^{1, b}$, Lijian Ji ${ }^{1, \mathrm{c}}$, Qinghua Ni ${ }^{1, \mathrm{~d}}$ \\ ${ }^{1}$ Army Officer Academy of PLA, Hefei , 230031, Anhui , China \\ awjxc1982@126.com bouo_zw@126.com dniqinghuapla@163.com
}

Keywords: Based on the information system, Artillery operation experiment platform, Design Abstract. Artillery operational experiment platform was designed from function and structure based on the information system. The functions of the platform is composed of five parts, which are role set, Guide and control, Command and control, Evaluation and decision, Multi-level integrated experiment. The structure of the platform is composed of data and model library, battlefield environment subsystem, Artillery operational evaluation subsystem and the result subsystem. The working process of the platform was analyzed, which included Experimental environment construction phase, Scenario editing phase, Implementation phase and Experimental evaluation phase. The models of platform were built to support system running, which including system architecture model, multi-resolution simulation model, complex battlefield environment model, scenario generate model, operational data model, etc.

\section{Introduction}

With a long period of construction, Artillery had built batch laboratories of equipment technology, equipment argumentation and equipment operational evaluation, with high degree of information. But it also lacks of experimental platform. It is difficult to support large-scale experiment to meet the needs of the future operation; it also can't meet the needs of Artillery operational experiment. Therefore, it is urgent to study of Artillery operational problems in laboratory environment, innovation and development Artillery operation theories, promote the formation of the Artillery operational capability based on information system.

\section{The overall design of Artillery operation experiment platform based on information system}

\section{Function design}

The platform provides operational scenario editor, experiment resource management, experiment environment, experimental guide control, the simulation operation support, the basic function such as operational effect evaluation of the experimental platform, to provide Artillery operational application experiment good usability, strong expansibility, build flexible system support. Its function mainly includes:

(1) The role set function. According to the experiment content, scale, person, it can set different seats and role assignment on different objects.

(2)Guide and control function. It includes the management and guidance control, monitoring, system control, system monitoring and display the basic function of battlefield situation.

(3)Command and control function. Through the computer network communication and combining with the battlefield situation, we can joint planning scheme, research, set decision of the battle. So, it complete the command and control action of the battlefield.

(4)Evaluation and decision function. It provides timely, accurate, comprehensive evaluation and award of the stage results of practice.

(5)Multi-level integrated experiment function. We regard the Artillery group, camp, even the multi-level commanders as experimental object, according to the command system, we build the multi-layer experimental environment. 


\section{Structure design}

The general structure of Artillery operation experiment platform can be divided into four parts, data and model library, battlefield environment subsystem, Artillery operational evaluation subsystem and the result subsystem of the experiment. The logical relationship between various systems is shown in Fig. 1. All subsystems are supported by environment RTI, we can achieve connectivity, communication, interoperability, in order to meet operation experiment under the environment of the operation software platform. Operational experiment platform software logic structure is shown in Fig. 2.

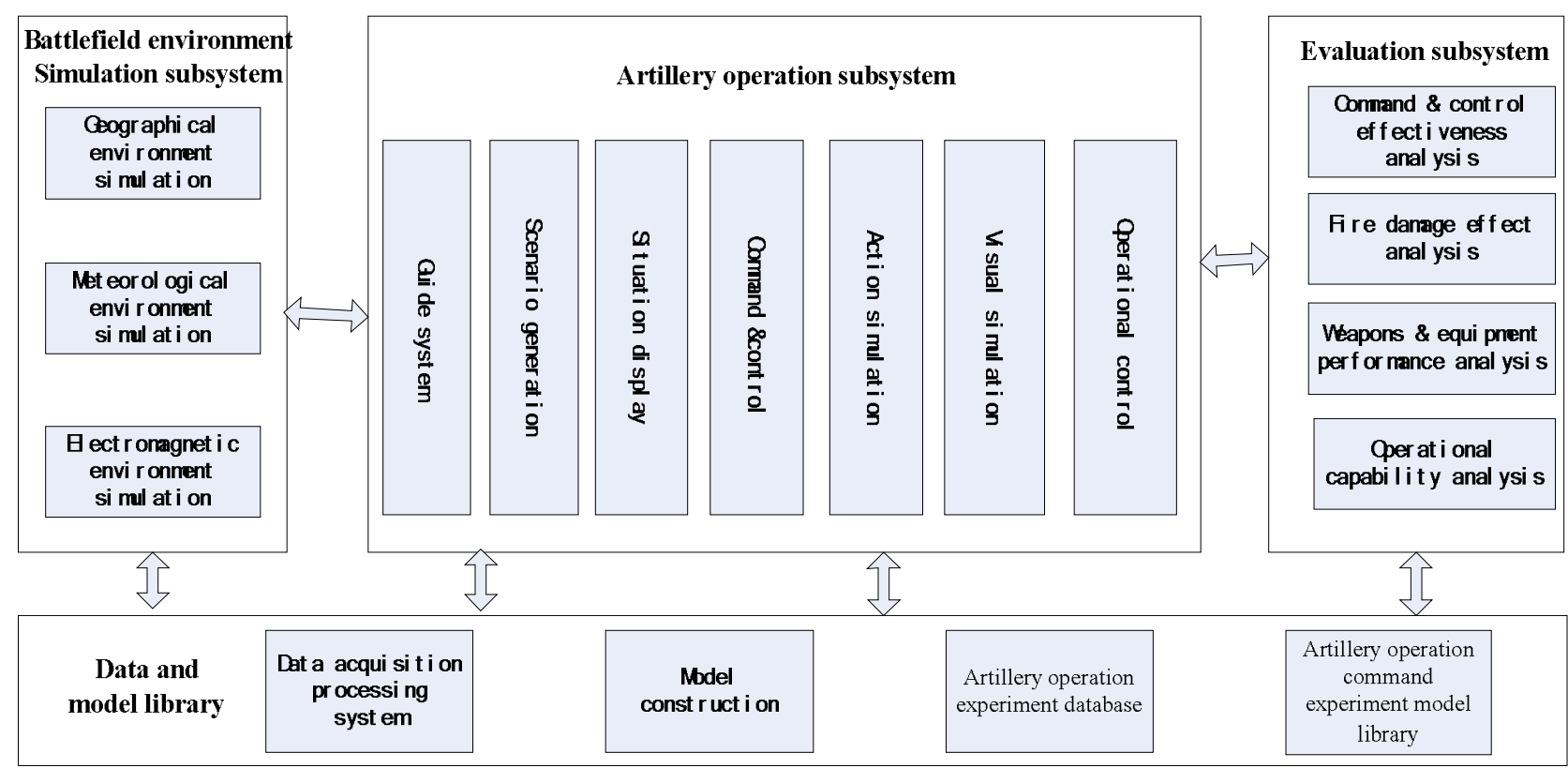

Fig. 1 The logical relationship of Artillery operation experiment platform

(1) Data and model library

This part is mainly collecting and processing data, making operation decision for the Artillery operation experimental, it provides data and the model service. It mainly provides Artillery operation experiment data processing and storage, model construction, model storage, data and model services, to manage the Artillery operational experiments data and model.

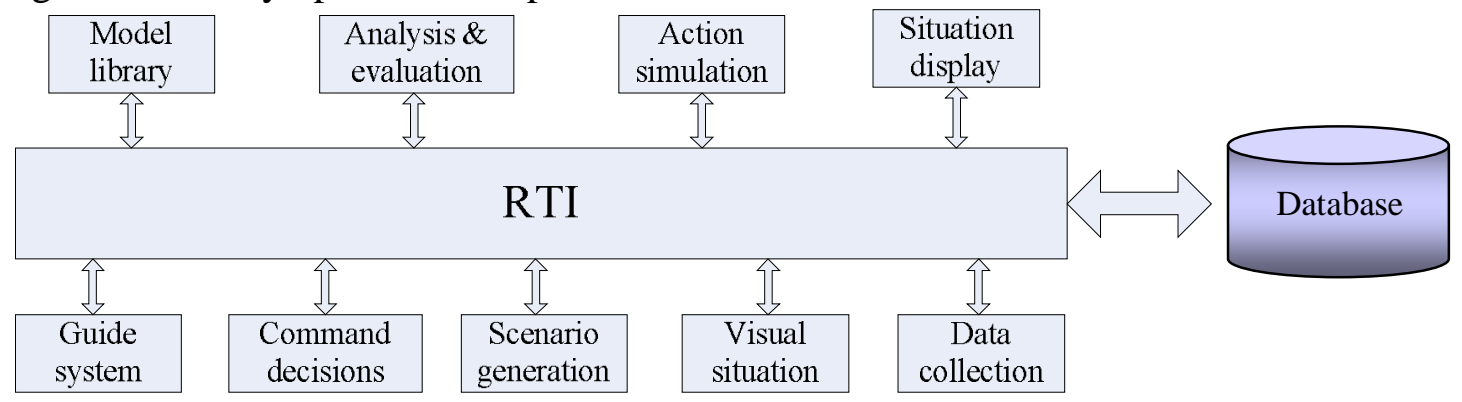

Fig. 2 software logical structure of Artillery operation experiment platform

(2) Battlefield environment Simulation subsystem

The battlefield environment simulation is mainly used to simulate Artillery battlefield environment, including geographical information, electromagnetic environment, weather information, it provides experiment data for the platform. It mainly realizes simulation geographical environment, geographical data information; it Simulates electromagnetic environment, provides electromagnetic environment's influence on the communication data, and the simulation of the communication quality; it simulates meteorological environment, provides meteorological environment impact on the Artillery operation action data. It supports a variety of editing and management of data field. 
(3) Artillery operation subsystem

The Artillery operation subsystem was built, mainly to organize the implementation and process management for the Artillery operation experiment. The subsystem is closely combined with operational experiment characteristics and law rules, through using advanced technology to build experimental environment, to ensure the centralized management and orderly operation of the Artillery operational experiments based on the basis of the laboratory network system construction achievements. The main implementation are guide, operational scenarios generated, making operation command decision, operation simulation, the battlefield situation display; operational control.

(4) Evaluation subsystem

This party is mainly used for the scheduled operations under the conditions of Artillery operations experiment data analysis and research, provides support to improve the operational capability. The main implementations are command and control effectiveness analysis, weapons and equipment efficiency analysis, fire damage effect analysis; battle command ability analysis.

\section{The workflow design of Artillery operational experiment platform based on the information system}

The working process of the Artillery operational experiment platform can be divided into four stages: the experiment environment to build, scenarios editing, experiment operation and the application effect evaluation.

\section{Experimental environment construction phase}

In accordance with the requirements for the experimental task decomposition, we can form experiment scheme. According to the experiment scheme requirements, we choose the appropriate software and hardware equipment, build the dynamic construction of experimental environment, when the existing resources cannot meet the requirement of the experiment, it needs to be developed or obtained from third parties, such as the lack of a weapon and equipment simulation model, we can develop the available resources from software platform.

\section{Scenario editing phase}

This phase mainly completes the scenario editor, preview and confirmation, update the weapon system of operation into basic data, to ensure the consistent with the simulation test environment.

\section{Implementation phase}

In the implementation phase, $t$ according to the scenario editing, the battlefield situation produce module dynamically generates battlefield environment and military activities information, drives the operation of all kinds of simulation model, organizes blue confrontation simulation system which is composed of weapons and equipment simulation model and component against, and record test data. In the process of advance, red and blue operation system against independently, through the guide and control function, we can adjust situation of battlefield in real-time, complete closed loop experiment.

\section{Experimental evaluation phase}

This phase is mainly performed by evaluation module. In the process of experiment, it collects and records the real-time data and results. After finished the experiment, we must carry on the experiment data archiving. According to the specific assessment tasks (including weapons and equipment effectiveness evaluation, operational application scheme evaluation and etc.), we can extraction and analysis experimental data, playback and analysis the experimental process.

\section{The model design of Artillery operation experiment platform based on information system}

\section{Architecture model}

Artillery operation experiment platform can adopt HLA (high level architecture) runtime support system KD - RTI and MAK - RTI, uses the object-oriented thought to realize each function of RTI, its 
composition is shown in Fig.3, it is mainly composed of guide, object model development tool (OMDT), scenario editor, the simulation engine, situation display, record and playback module.

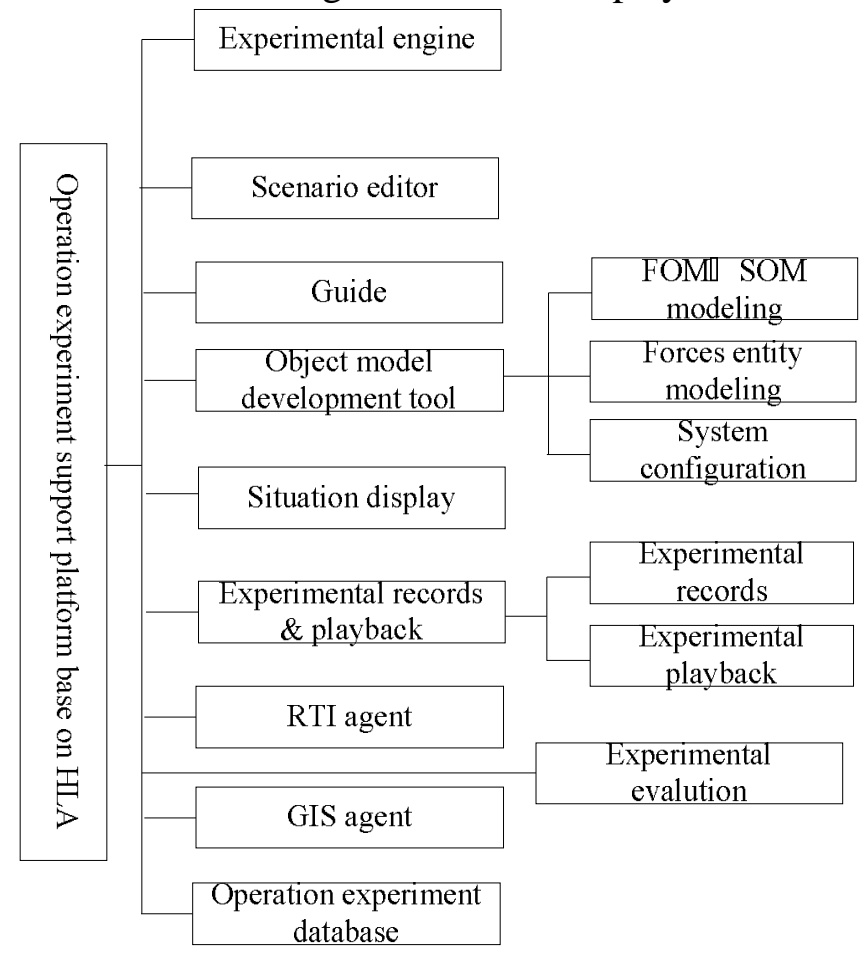

Fig. 3 The support environment of Artillery operational experiment operation

\section{Multi-resolution simulation model}

The resolution of the operation simulation, which is to describe the detail between the real world and simulation object: the granularity. Usually it adopts fixed resolution model, such as the size of campaign usually is pre-determined army, division and regiment levels resolution, but that is not easy to obtain the local process simulation of further refinement, in some important season, commander hopes enlarge the Artillery group operation, which requires the action refinement to the Artillery battalion and battery unit level, here, we have to adopt variable resolution simulation.

The key technology of multi-resolution modeling is how to keep the consistency between different resolution model, guarantees their mutual transformation seamless. When commander enlarge the Artillery group, it converts to the multi-resolution model, which the data and action resolution enlarge to the unit level, and other parts of the battlefield is still keep the original resolution, which requires the high-resolution and other parts of the original resolution under the condition, here also need a seamless restore to the original resolution under state of operation simulation, and the strategy is consistent.

\section{Complex electromagnetic environment model}

The battlefield electromagnetic environment is characterized by a certain battle space state of all kinds of electromagnetic signals. Electromagnetic environment radiation source can be divided artificial electromagnetic radiation source and natural electromagnetic radiation source. For artificial electromagnetic radiation includes deliberately radiation and no intention radiation. The research of battlefield electromagnetic environment is aimed at man-made electromagnetic information useful and targeted electromagnetic interference. In order to build and set out distinct, reliable battlefield electromagnetic environment, to adapt the complex electromagnetic environment of operation and training, it needs to build the correct battlefield electromagnetic model. The overall framework of the electromagnetic environment simulation model is shown in Fig.4. The main models are: signal model, the antenna model, simulation model of signal propagation and the corresponding algorithms library. 


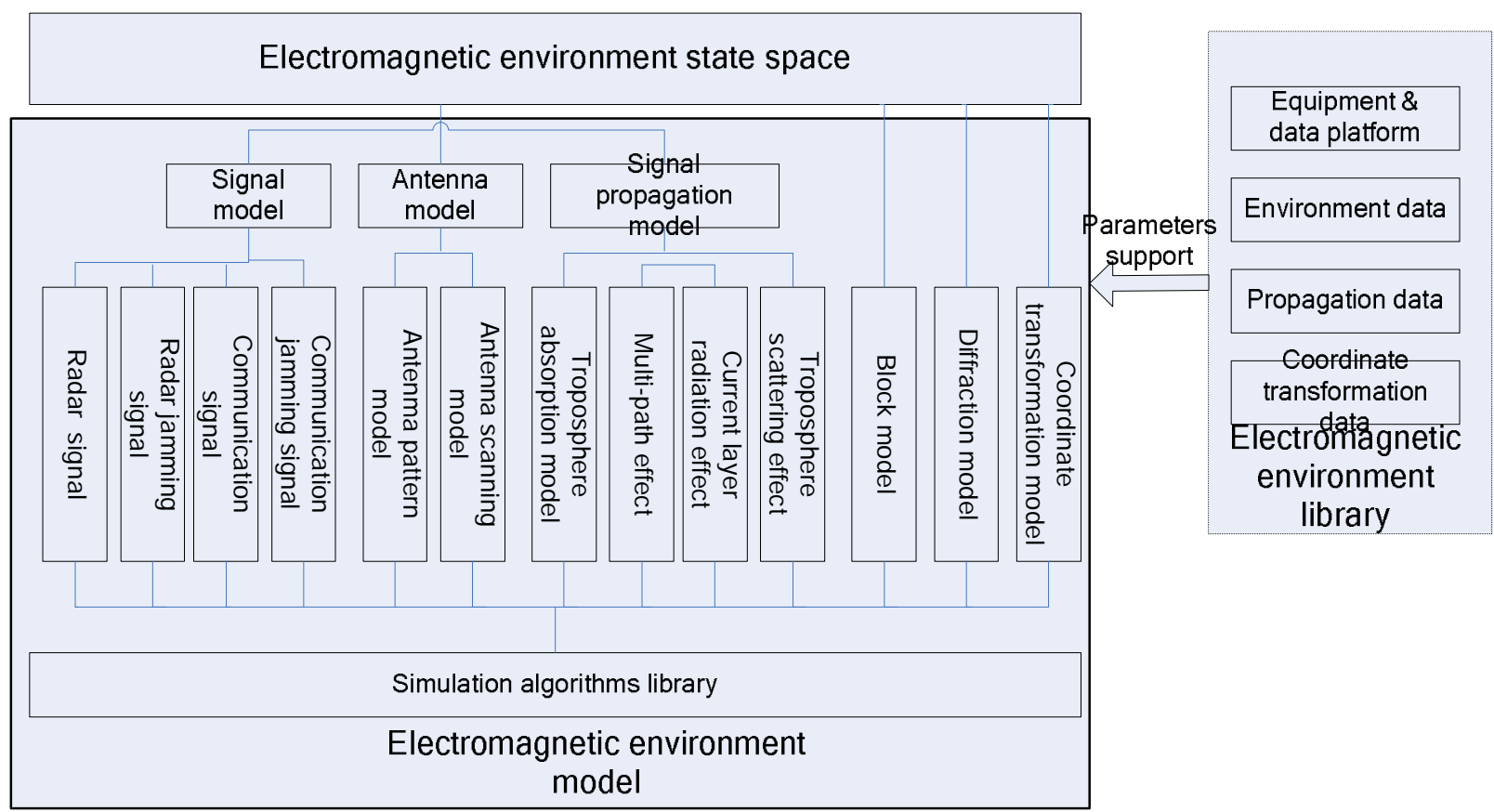

Fig. 4 The overall framework of electromagnetic environment simulation model

(1) Signal model. In order to build real battlefield electromagnetic environment, source network system must be considered. It mainly includes the communication network and radar net.

(2) Antenna model. According to the scanning pattern build antenna model.

(3)Signal propagation model. With the aid of this kind of model, we can quantify and analysis the electromagnetic wave in the air.

(4) Simulation algorithms library. It mainly includes coordinate transform, signal generation, signal processing, data processing and other basic math functions.

\section{Scenario generation model}

At the beginning of the operational experiments, we need a basic operational experiment condition, the initial conditions generally given by operational scenario data, it determines the basic conditions and the corresponding constraints of the operational experiments, including the simulation of the operational entity and the environment, the basic attributes of each entity, the entity dynamic properties determine the system state, and the use of plans, rules, agreed to limit the simulation process and constraints. To simplify the experiment content, determines the scope and process of the operational experiment, gives the basic background and premise condition, making operational experiment aim and object, setting simulation boundary conditions and constraints, are done through operational scenarios.

Operational scenario design generally includes background generation and battle plans fiction, and action plan, etc. Operational scenario background generation is a problem of the initial state background data loading, including unit organization, natural environment, social environment, the physical system and other aspects data. Battle plan fiction is the description of operational activities, which is in the initial condition under the operational condition of constraint. Action planning is to convert military scenarios to a simulation experiment script.

\section{Operational data model}

Operational data is the key factor of operational reliability experiment system, it mainly includes the data about the operational deployment of both sides, the target data, weapon system, operation action data, operational security data and evaluation criteria data, etc. The purpose of operational data is to provide data for the Artillery operation command experiment, as well as the situ experimental data storage place, it is not only the experiment system of data source, but also the destination of experimental system. Therefore, operational data construction including experimental data construction and the experimental conclusion construction. The application process is shown in Fig.5. 


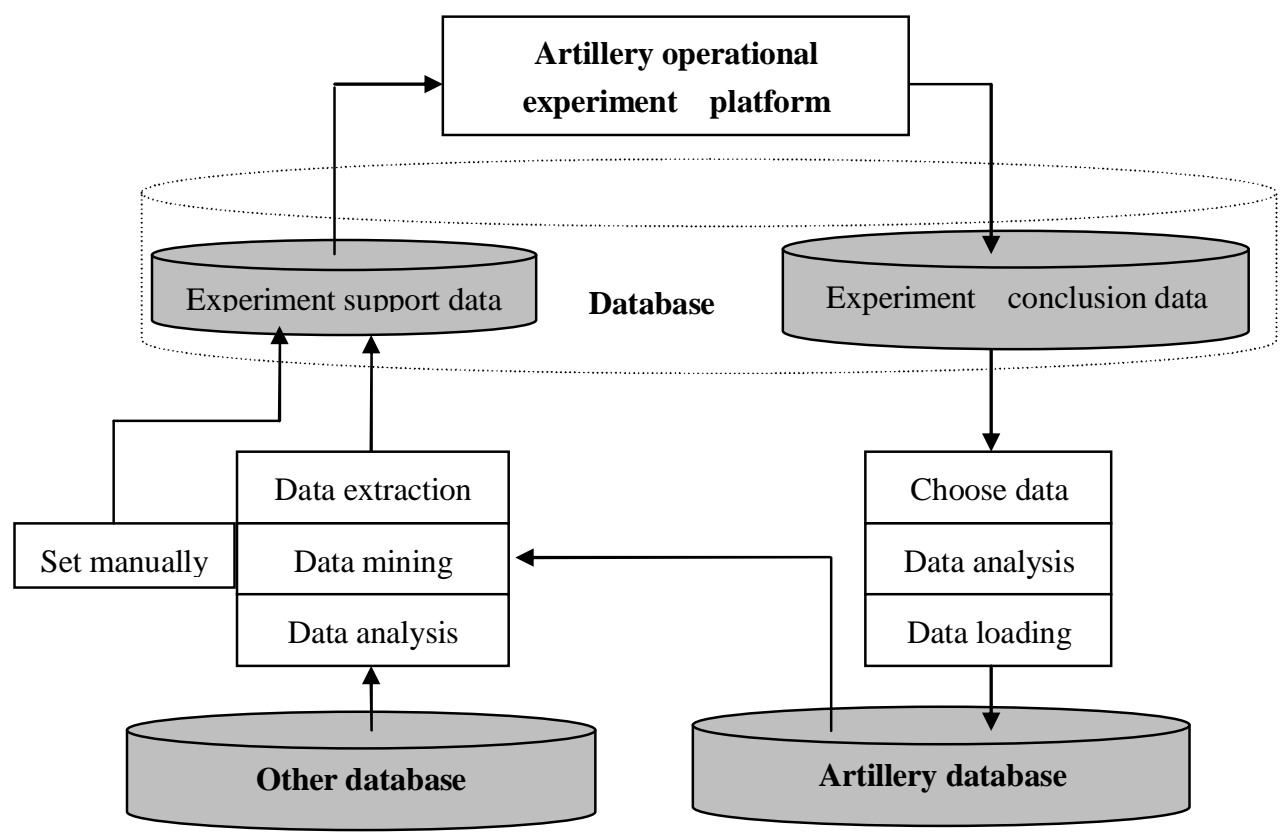

Fig. 5 Data application process

\section{Conclusion}

Carrying out the Artillery operation experimental research based on Artillery operational experiment platform, is to build experimental environment under the background, and then puts into experiment platform for experiment, uses data analysis method, build reasonable appraisal indicators, gets certain reference value for Artillery operation experimental data and results.

\section{References:}

[1] Zhongwei Guo etc. Artillery operation experimental model [J]. Ordnance industry automation, 2013, 32 (9)

[2] Yuhua Cao etc. Operational experiment theory and technology [M]. Beijing: National defence industry press, 2013

[3] Daolei Zhou Jiang Zhu. Methods of innovation and experiment [M]. Beijing: Military science press, 2014

[4] Richard Kass . The Logic of Warfighting Experimentation[C] . Understanding Joint Warfighting Experiments . 10thICCRTS , 2005

[5] Andndrew G Loerch , Larry B Rainey . Methods for conducting Military Operational Analysis[M] . Military Operations Research Society , 2007

[6] Shadish , Cook , Campbell . Experimental and Quasi-experimental Designs for Generalized Causal Inference[M] . Boston:Houghton Mifflin , 2002

[7] Sherman Kenneth B . US Navy Global Hawk Completes Wargame[J] . Journal of Electronic Defense , 2006 , 2(29) 
[8] Wolff Jason . Wargame, Modeling and Simulation[J] . Air Force Journal of Logistics , 2009 , 2(33) 